\title{
The ATLAS ITk Strip Detector. Status of R\&D
}

\author{
Carlos García Argos, on behalf of the ATLAS ITk Strip Collaboration
}

CERN, 1211 Geneva, Switzerland

\begin{abstract}
While the LHC at CERN is ramping up luminosity after the discovery of the Higgs Boson in the ATLAS and CMS experiments in 2012, upgrades to the LHC and experiments are planned. The major upgrade is foreseen for 2024, with a roughly tenfold increase in luminosity, resulting in corresponding increases in particle rates and radiation doses. In ATLAS the entire Inner Detector will be replaced for Phase-II running with an all-silicon system. This paper concentrates on the strip part. Its layout foresees low-mass and modular yet highly integrated double-sided structures for the barrel and forward region. The design features conceptually simple modules made from electronic hybrids glued directly onto the silicon. Modules will then be assembled on both sides of large carbon-core structures with integrated cooling and electrical services.
\end{abstract}

Keywords: ATLAS, HL-LHC, tracker, silicon

\section{Introduction}

The current ATLAS Inner Detector (ID) [1] will be replaced by an all-silicon Inner Tracker (ITk) for the High Luminosity LHC, between 2024 and 2026. The upgrades on the accelerator complex will allow the HL-LHC to deliver up to $3000 \mathrm{fb}^{-1}$ by 2037 , through an increase in the instantaneous luminosity of five times with respect to the current LHC. As a consequence, the experiments will have to cope with up to 200 interactions per crossing [2].

These increases pose a challenge to the trackers of ATLAS and CMS, both in the capability to resolve the tracks and regarding radiation damage. New tracker designs will be installed, with higher granularity and more radiationtolerant sensors that can withstand the increased fluence.

\section{The ATLAS Tracker Upgrade for HL-LHC}

The baseline layout of the ITk from the Letter of Intent [3] is shown in Figure 1. The final layout is still under discussion, with ongoing studies on the possibility to extend the pseudorapidity coverage of the tracker [2].

The number of channels on the pixel detector will increase from around 80 million in the current design to around 600 million and the strips tracker will have around 70 million channels compared to the current 6 million of the SCT.

Material-wise, the tracker has to fit in the space left by the ID, inside the solenoid, including all the services: cooling pipes and powering and data cables. Figure 2 shows the simulation of the ITk material.

Email address: carlos.garcia.argos@cern.ch (Carlos García Argos, on behalf of the ATLAS ITk Strip Collaboration)

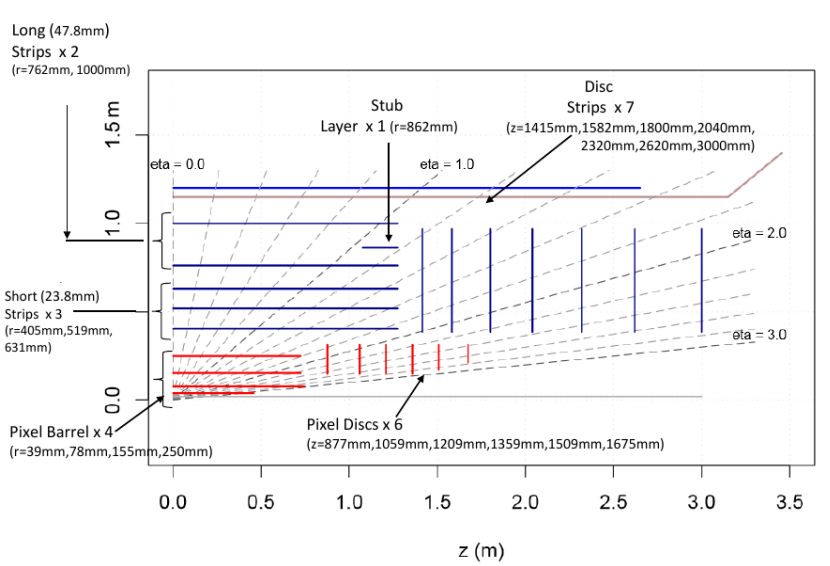

Figure 1: Baseline layout of the ATLAS ITk.

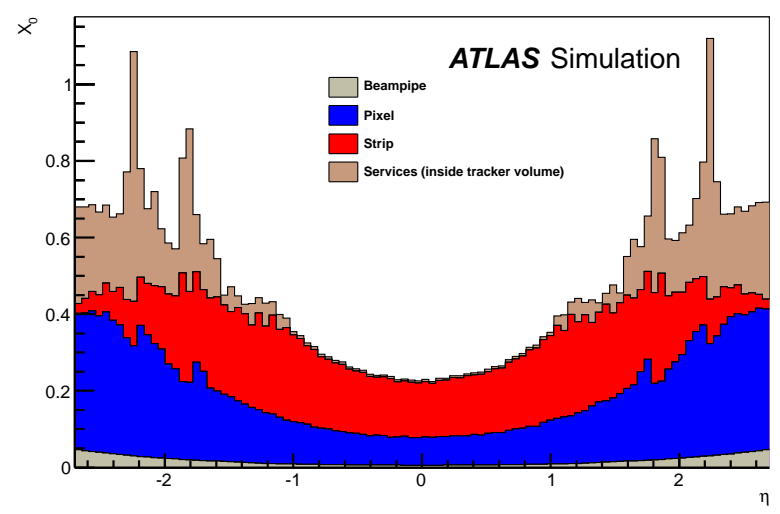

Figure 2: Simulation of the material in the ITk. 


\subsection{Strips Sensors and Modules}

The strips detector is composed of silicon based modules mounted on supporting structures, called staves in the barrel and petals in the endcap discs. The purpose of these structures is to have multi-module support, modularity and integration of cooling and read-out electronics.

Read-out chips are glued on a flex hybrid circuit. The hybrid is then glued on the silicon sensor and wirebonds connect the sensor strips to the chips front-end inputs.

\subsection{Read-out Electronics}

The read-out electronics of the sensors employs the ABC130 (ATLAS Binary Chip in a $130 \mathrm{~nm}$ process). This chip processes all the signals coming from 256 strips and is optimised for strips up to $6 \mathrm{~cm}$ long with $25 \mathrm{~ns}$ shaping time. The expected noise after irradiation is below $1000 e^{-} \mathrm{ENC}$.

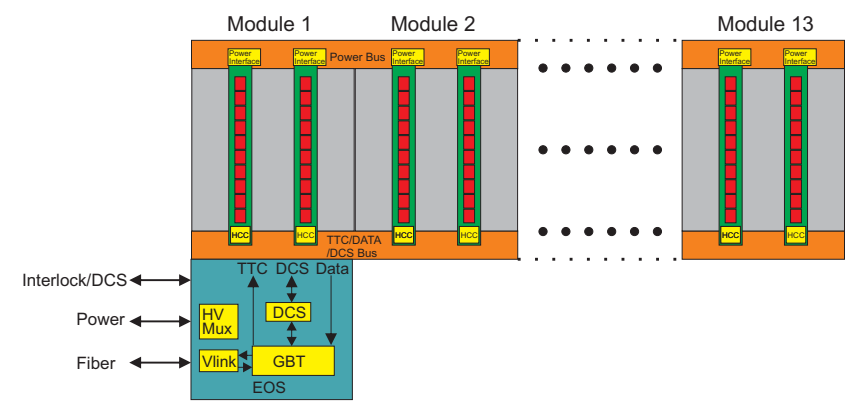

Figure 3: Read-out topology of the ABC and HCC chips.

The ABC130 sends data to the next chip in the chain, the Hybrid Control Chip (HCC), at a rate of up to $320 \mathrm{Mbps}$, then the HCC sends the data at up to $640 \mathrm{Mbps}$ to the End of Structure (EoS). The End of Structure card aggregates various functions such as DCS, power and data read-out. The data is sent off-detector at up to $8.86 \mathrm{Gbps}$ through the Versatile Link PLUS [4]. Figure 3 shows the signals path on a stave.

\subsection{Powering Scheme}

With the increase in the total number of channels, and despite a reduction in the power consumption per channel, more total power is required for the ITk than for the current ID. In addition, there is no space for extra cables from the counting rooms to the cavern, and adding more cables would lead to extra material. Therefore, a more efficient way to provide power to the modules is required for the ITk.

The baseline for the strip detector is DC to DC conversion, with off-detector power supplies at a higher voltage, reducing the current on the cables and the ohmic losses [5]. On-detector, buck DC-DC converters lower the voltage to the required by the on-detector electronics. This requires the design of radiation hard DC-DC converters [6]. The DC-DC powering scheme is shown in Figure 4.

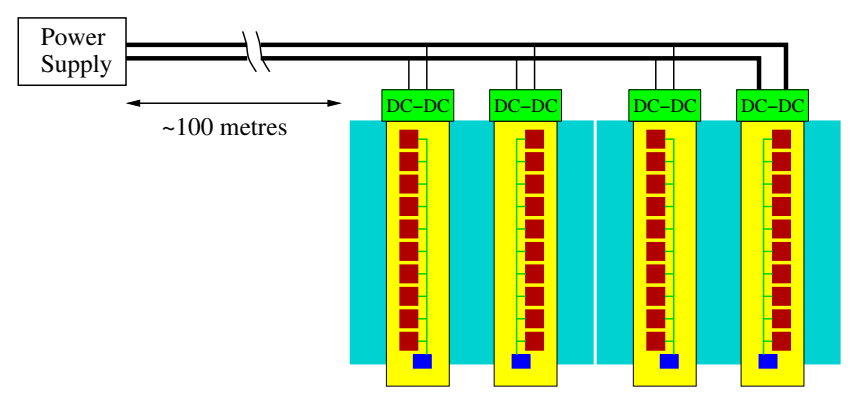

Figure 4: Powering with DC-DC conversion.

In addition, multiplexing of the high voltage sensor bias lines is preferred, since multiple modules could be biased through a single cable. This also requires radiation hard high voltage transistors that can switch voltages up to $1000 \mathrm{~V}$, a programme which is still ongoing.

\subsection{Staves and Petals Concept}

The staves and petals for the ITk are double-sided structures, aimed at low material, high rigidity and modularity. They provide local support, cooling and electrical services to the modules. In the barrel region, they are straight with 14 rectangular modules, whereas in the endcap region the geometry makes the modules differently sized and shaped. Drawings of a stave and a petal with their components are shown in Figure $5[8,9]$.

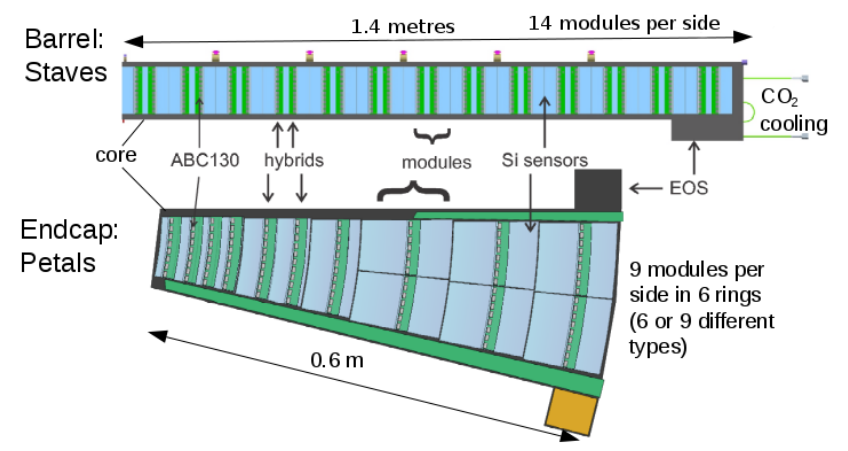

Figure 5: Drawings of a stave and a petal.

The assembly is simplified for mass production, with the modules glued on a bus tape which is co-cured on a carbon core structure. The services are multiplexed and shared among the modules on a stave/petal. The EoS card is the interface between the staves/petals and the outer world.

Both structure types are designed to be insertable at the end of the support structures. An example of the barrel insertion is shown in Figure 6. This modularity eases the final assembly of the barrel and endcaps. There are a total of 472 staves and 448 petals to be mounted in the baseline layout. This type of assembly also allows for earlier testing of larger global structures. Finally, the services are connected at the end of the structures, which leads to less material inside the tracker. 


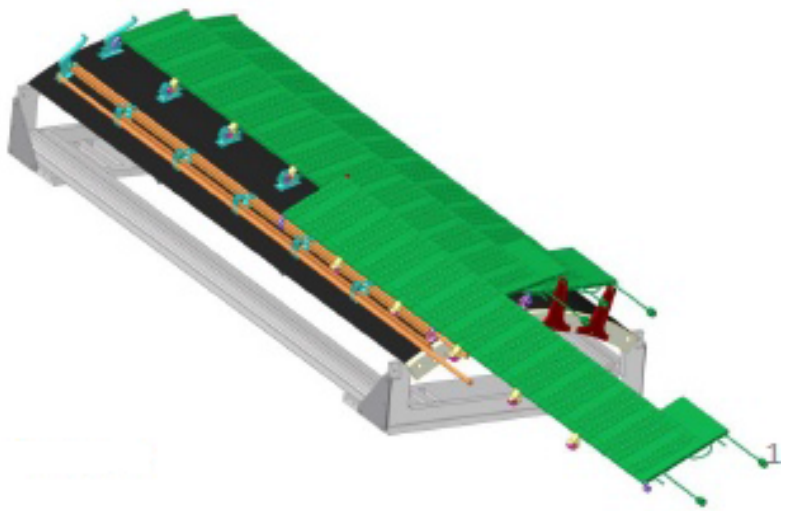

Figure 6: Barrel end insertion.

\section{Status of Prototyping and Testing}

\subsection{Sensors}

The silicon sensors will have to withstand a maximum fluence of $2 \times 10^{15} \mathrm{n}_{\mathrm{eq}} \mathrm{cm}^{-2}$, with a safety factor of 2 . Because of this, $\mathrm{p}-\mathrm{in}-\mathrm{n}$ sensors are not suitable. The baseline for the ITk strip detector are n-in-p Float Zone sensors with p-stop as strip isolation. Because they are fabricated in a single-sided process, they are easy to handle and more foundries are available, reducing cost with respect to double-sided processes.

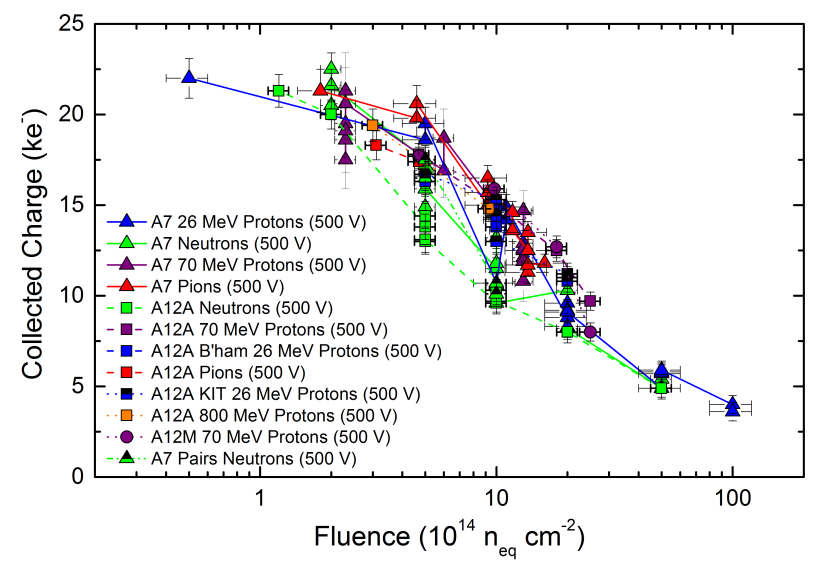

Figure 7: Collected charge for ATLAS07 and ATLAS12 sensors.

The sensors for the barrel are $10 \mathrm{~cm} \times 10 \mathrm{~cm}$, and for the endcap they have different shapes and sizes due to the more complicated structure. The barrel sensors have four rows of 1280 active strips with a length of $23.82 \mathrm{~mm}$, a pitch of $75 \mu \mathrm{m}$, and the thickness is around $300 \mu \mathrm{m}$.

Two pre-production series, called ATLAS07 and ATLAS12, have been tested and irradiated. Figure 7 shows the collected charge of both, at $500 \mathrm{~V}$ for minimumionising particles for n-in-p FZ sensors, after proton, neutron and gamma irradiation, beta source tests and 80 minutes annealing at $60^{\circ} \mathrm{C}[2]$.
As the plot shows, both types of sensors have a similar performance, with an observed signal of around 12000 electrons at $1 \times 10^{15} \mathrm{n}_{\mathrm{eq}} \mathrm{cm}^{-2}$.

\section{2. $250 \mathrm{~nm}$ Programme}

The initial prototyping of the ITk started with $250 \mathrm{~nm}$ ASICs, named ABCn25, CMOS based, as an evolution of the current SCT read-out chip [7]. The programme finalised with the construction and testing of a DC-DC powered stave with 12 modules on one side, a serially powered stave with 12 modules on one side and two DC-DC powered, double-sided petalets.

The stave with DC-DC powering showed better performance than the serially powered one, leading to DC-DC conversion being the current baseline.

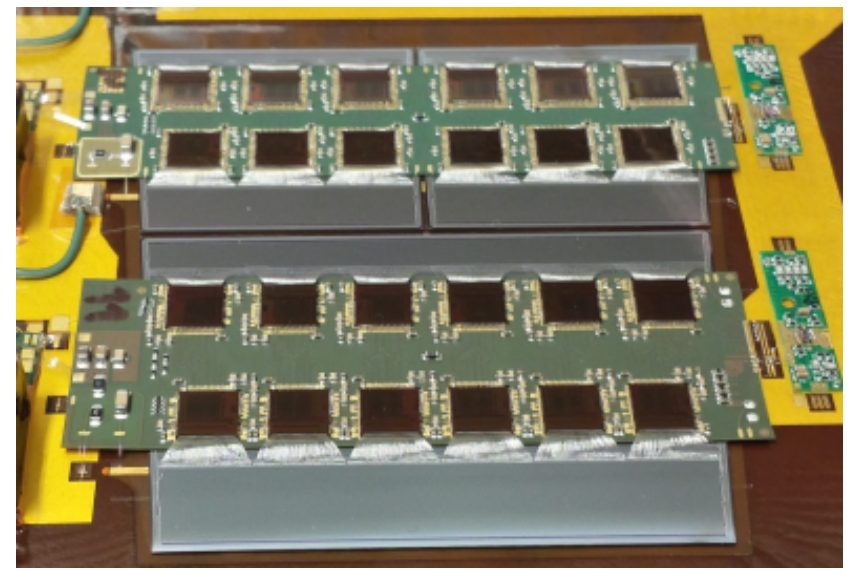

Figure 8: Petalet prototype.

For the petalet programme, the goal was to have a reduced version of the petal using differently shaped modules as proof-of-concept. The design of the petalet was such that it allowed testing the region where the petal splits in two sensors $[10,11]$. One of such petalets is shown in Figure 8 . There are two options to read-out the split sensors: one single hybrid for both or a split hybrid, reading out each sensor with one. The performance of double-sided petalets was the same for both approaches, but the split option was chosen for easier handling and better placement accuracy of the modules.

\section{3. $130 \mathrm{~nm}$ Programme}

The first ABC130 based hybrids, both barrel and endcap, were ready recently. They are shown in Figures 9 and 10, respectively. The chip read-out has been tested at $160 \mathrm{Mbps}$ data rate with an $80 \mathrm{MHz}$ clock. The first $\mathrm{HCC}$ read-out has also been tested recently and the first hybrids are in the process of being finalised.

Transmission tests on long bus tapes at a rate of $640 \mathrm{Mbps}$ are planned, and institutes are getting ready to build and test ABC130 and HCC based hybrids and modules. 


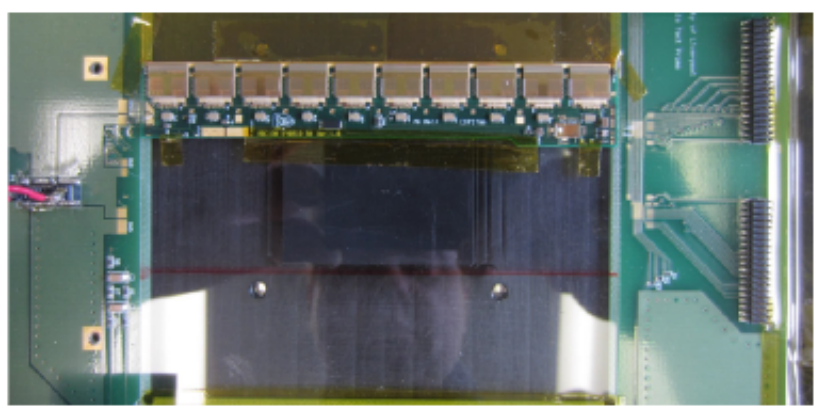

Figure 9: First ABC130 strip barrel module.

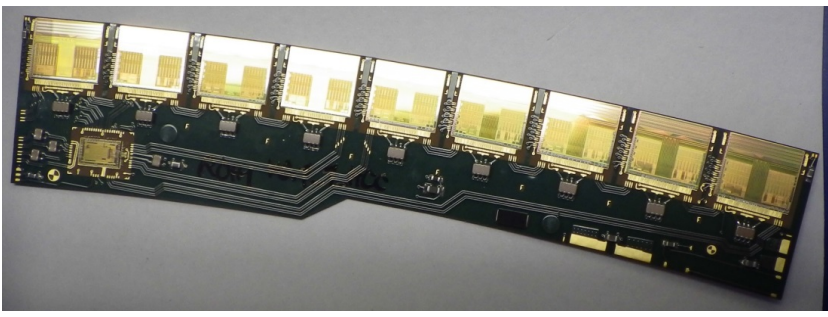

Figure 10: First ABC130 strip endcap hybrid.

To complement the electrical tests of hybrids and modules, testbeams have been performed at DESY. More testbeams will be done both at DESY and at CERN, with the goal of characterising the read-out chips and the detectors.

\section{Towards Mass Production}

Production of the ITk sensors, hybrids and modules should start around 2017 or 2018. The number of items to be accounted for will pose a challenge for production, quality assurance and quality control. Around 300,000 ABC130 and HCC chips will be needed in the installed tracker, for over 30,000 hybrids, more than 10,000 barrel modules and over 8,000 endcap modules.

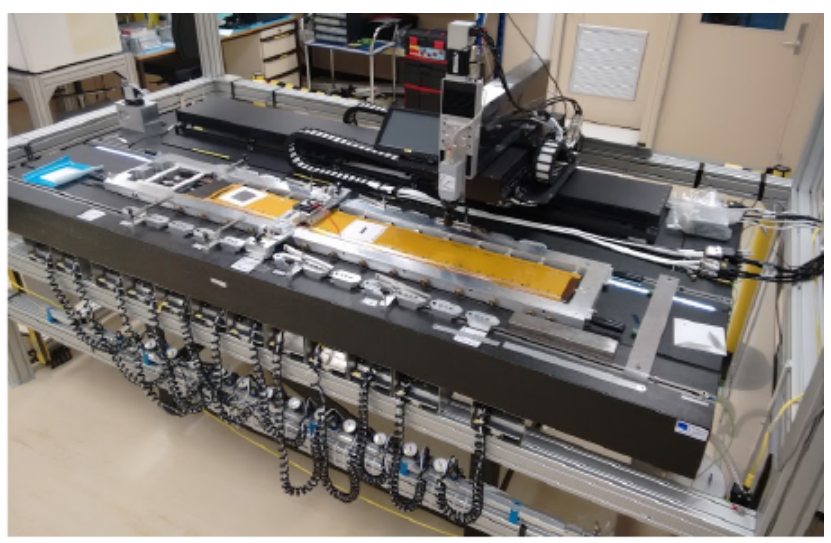

Figure 11: Module placement machine for staves.
The mounting of modules on staves and petals has to be done with high precision, for which placement machines will be used, like the one shown in Figure 11.

\section{Conclusions}

The R\&D of the ATLAS ITk Strips detector is well advanced, with some details such as the final layout still being studied. The sensors have already been selected and characterised, with the read-out chips being fully understood.

Functional modules and larger structures will arrive soon for the first tests. The development of system tests and the data acquisition system is also advancing well.

The construction of the modules and larger structures will take place at multiple sites, with special attention to quality control and quality assurance.

\section{References}

[1] ATLAS Collaboration, The Expected Performance of the ATLAS Inner Detector. ATL-PHYS-PUB-2009-002, 2008.

[2] ATLAS Collaboration, ATLAS Phase-II Upgrade Scoping Document, CERN-LHCC-2015-020. LHCC-G-166, 2015.

[3] ATLAS Collaboration, Letter of Intent for the Phase-II Upgrade of the ATLAS Experiment , CERN-LHCC-2012-022. LHCC-I023, 2012.

[4] Vasey, F. et al. The Versatile Link PLUS Project, 2015.

[5] Affolder, A. et al. DC-DC converters with reduced mass for trackers at the HL-LHC, JINST 6 (2008).

[6] Faccio, F. et al. FEAST2: A Radiation and Magnetic Field Tolerant Point-of-Load Buck DC/DC Converter, REDW 2014.

[7] Kaplon, J. The ABCN front-end chip for ATLAS Inner Detector Upgrade, TWEPP 2013.

[8] Díez, S. Silicon strip staves and petals for the ATLAS Upgrade tracker of the HL-LHC, NIM A 699 (2013).

[9] Díez, S. et al. A double-sided, shield-less stave prototype for the ATLAS Upgrade strip tracker for the High Luminosity LHC, JINST 9 (2014).

[10] Wonsak, S. A forward silicon strip system for the ATLAS HLLHC upgrade, RESMDD 2012.

[11] Kuehn, S. The Phase-2 Upgrade of the Silicon Strip Tracker of the ATLAS experiment, Vertex 2013. 